\title{
Die Internationale Zeitschrift für Rechtslinguistik - „JLL“
}

\author{
Hanjo Hamann*
}

Eine wichtige Nachbardisziplin der Rechtswissenschaft ist die Sprachwissenschaft. Nachdem Juristen sich schon lange für die spezifischen Eigenheiten der Rechtssprache interessieren und sich immer wieder anekdotisch damit befassen, kommt es in den letzten Jahren auch auf der Ebene der wissenschaftlichen Methoden und Erkenntnisinteressen zu einer systematischen Annäherung der Wissenschaft vom Recht (als performativem Sprachgebrauch) und der Wissenschaft von der Sprache (als pragmatischem Zeichengebrauch). Diese Annäherung, die in Deutschland spätestens 1984 mit der Gründung der Heidelberger Arbeitsgruppe der Rechtslinguistik begann, ist mittlerweile zur Rechtslinguistik als einer gefestigten Transdisziplin mit institutionellen Strukturen gereift. Eine dieser Institutionen ist die internationale Zeitschrift für Rechtslinguistik, das International Journal of Language \& Law (JLL), das seit 2012 als Platin-Open-Access-Zeitschrift erscheint.

\section{A. Das International Journal of Language \& Law (JLL)}

Die Fachdisziplin Rechtslinguistik, ${ }^{1}$ die aus Parallelbewegungen vor allem in den USA und Deutschland, aber auch der Schweiz, Spanien, Polen und weiteren Ländern hervorgegangen ist, ${ }^{2}$ wurde erstmals im Jahr 2007 institutionalisiert. Die damals von den US-amerikanischen Rechtsprofessoren Lawrence M. Solan und Peter M. Tiersma gemeinsam mit dem deutschen Sprachwissenschaftler Dieter Stein gegründete internationale Vereinigung für Rechtslinguistik, International Language and Law Association (ILLA), beschloss mit ihrer Tagung in Los Angeles im September 2019 das dreizehnte Jahr ihres Bestehens. Seit acht Jahren verfügt sie mit dem JLL über ein eigenes wissenschaftliches Publikationsorgan.

JLL ist eine englischsprachige Online-Zeitschrift mit doppelt-verblindeter Kreuzbegutachtung (Peer Review), die „ein Forum zur Erforschung der Wechselwirkungen von Sprache und Recht in all ihren Facetten“ bieten will, „von theoretischen Ansätzen bis zu praktischen Problemen “. ${ }^{3}$ Die Zeitschrift versteht sich als PlatinOpen-Access, erscheint also kostenfrei für Autoren und Leser, wird von Wissen-

* Dr. Dr. Hanjo Hamann ist Senior Research Fellow am Max-Planck-Institut zur Erforschung von Gemeinschaftsgütern (Bonn). Der Text beruht streckenweise auf einem englischsprachigen Bericht (JLL 2019, S. 1), bei dessen Übertragung ins Deutsche Neele Hoffmann dankenswerte Unterstützung und Thomas Holzhausen Korrekturhilfe geleistet hat.

1 Überblick bei F. Vogel, Rechtslinguistik: Bestimmung einer Fachrichtung. In Felder \& Vogel (Hrsg.), Handbuch Sprache im Recht, 2017, S. 209.

2 Vgl. F. Vogel/H. Hamann/D. Stein/A. Abegg/L. Biel/L. Solan, Begin at the Beginning: Lawyers and Linguists Together in Wonderland, The Winnower 2016, Nr.4919, online unter doi.org/10.15200/ winn.148184.43176; zu den deutschen Wurzeln H. Hamann/F. Vogel, Evidence-Based Jurisprudence meets Legal Linguistics. Unlikely Blends Made in Germany, BYU L. Rev. 43 (2018), S. 1473.

3 JLL-Website (LanguageAndLaw.eu), archiviert unter web.archive.org/20190304152349/languageandl aw.eu. 
schaftlern in Eigenregie geleitet und lizensiert sämtliche Inhalte unter einer liberalen CC-BY-Lizenz. Das unterscheidet sie von anderen Zeitschriften im selben Forschungsgebiet, etwa der beinahe gleichnamigen, aber zwei Jahre jüngeren Language and Law=Linguagem e Direito (LLLD) aus Portugal oder der Kölner Zeitschrift für Europäische Rechtslinguistik (ZERL), die zwar ebenfalls kostenfrei online erscheinen, deren Lizenzen aber auf unkommerzielle unbearbeitete Verwendungen beschränkt sind (CC BY-NC-ND), ${ }^{4}$ was nach weithin vertretener Ansicht die Voraussetzungen des Open Access bestenfalls beschränkt erfüllt. ${ }^{5}$

Der folgende Bericht über die Gründung und weitere Entwicklung der Zeitschrift JLL orientiert sich an den drei Entwicklungsetappen, die das JLL in den ersten sieben Jahren seines Bestehens durchlaufen hat (B-D) und deutet Entwicklungsperspektiven unter dem neuen Schriftleiter Aleksandar Trklja an (E).

\section{B. Das erste Triennium (2012-2014): „Eine Welt, die immer stärker zusammenwächst“}

Ebenso wie die ILLA wurde auch ihre Zeitschrift von den Rechtslinguisten Tiersma, Solan und Stein gegründet. In ihrem knappen Auftakt-Editorial zum ersten Jahrgang formulierten die Gründungsherausgeber den theoretischen Ausgangspunkt des JLL wie folgt:

„Die Gesetze einer Gesellschaft bestehen nicht, wo sie nicht erörtert und ausgehandelt werden. Die ,Bedeutung' und der Bedeutungswandel von Rechtskonzepten, ebenso wie die ,Interpretation' von Begriffen, sind hauptsächlich eine Art sprachwissenschaftlicher Analyse, die mit hartnäckigen Schwierigkeiten einhergeht, zu denen Vagheit, Mehrdeutigkeit und Genauigkeit ebenso gehören wie schlichte Verständlichkeit ." "6

Trotz des pragmatischen Grundverständnisses, das im ersten Satz mitschwingt, wollten die Gründungsherausgeber die Zeitschrift nicht auf eine bestimmte theoretische Denkschule beschränken. Stattdessen entwarfen sie ein umfassenderes, integratives Leitbild für das JLL:

„In einer Welt, die immer stärker zusammenwächst in Wirtschaft, Recht und Kommunikation, ist es nur angemessen, dass die Forschung zur Sprache im Recht alle Schnittstellen zwischen diesen Disziplinen abdeckt. "7

Dieses integrative Leitbild, das angesichts politischer Verwerfungen noch heute kaum zeitgemäßer sein könnte, wurde von den Gründungsherausgebern sogleich auch ganz praktisch ins Werk gesetzt: Für den ersten Jahrgang ihrer Zeitschrift ver-

4 Zur ZERL web.archive.org/20190304151234/zerl.uni-koeln.de/redaktionelle-richtlinien.html, zur LLLD web.archive.org/20190304151813/ojs.letras.up.pt/index.php/LLLD/about/editorialPolicies.

5 Dazu H. Hamann, in diesem Heft, S. 108.

6 L. Solan/D. Stein/P. Tiersma, Introducing "Language \& Law", JLL 2012, S. 1, abrufbar unter doi.org/10.14762/j11.2012.001.

7 Solan/Stein/Tiersma, Introducing (Fn. 6). 
pflichteten sie einen Gastherausgeber (guest editor) aus einem weiteren europäischen Land: Jaap Baaij, damals noch an der Universität Amsterdam, versammelte für den ersten JLL-Jahrgang eine beeindruckende Riege internationaler Autoren, die aus ihren jeweiligen Arbeitsgebieten über verschiedene Facetten von Mehrsprachigkeit, Recht und Sprachpolitik reflektierten.

Was die technische Umsetzung der Zeitschrift angeht, war das JLL seit Gründung 2012 ausschließlich im Internet unter der Domain www.LanguageAndLaw.de erreichbar. Die deutsche Domainendung entsprach der Nationalität des ersten Schriftleiters Prof. Dr. Dieter Stein von der Universität Düsseldorf. Wohl auch aufgrund dieser Anbindung nutzte die Zeitschrift ursprünglich das Publikationssystem der deutschen Digital Peer Publishing Initiative (DiPP, dipp.nrw.de). Dabei kam deren proprietäre Open Access Lizenz zum Einsatz (DPPL), ${ }^{8}$ für die persistente Adressierung der Zeitschriftenbeiträge wurden Uniform Resource Names (URN) genutzt. $^{9}$

Abb. 1: Screenshot der JLL-Website auf der DiPP-Plattform (2012-2015).

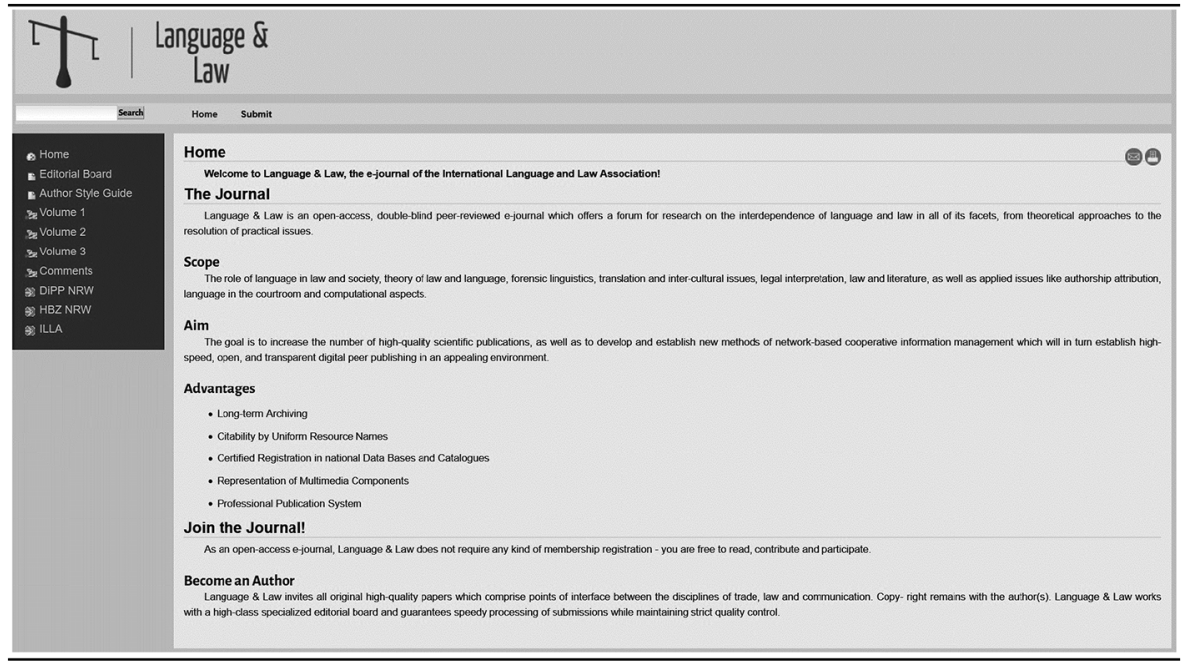

Screenshot vom 16. Januar 2016, kurz vor der Umstellung auf OJS (dazu gleich Abschnitt 4).

8 A. Metzger/T. Jaeger, Digital Peer Publishing Lizenz (v 3, de) @ 2004-2008 Ministerium für Innovation, Wissenschaft, Forschung und Technologie des Landes Nordrhein-Westfalen, archiviert unter web.archive.org/20190304222117/hbz-nrw.de/produkte/open-access/lizenzen/dppl/dppl/DPPL_v 3_de _11-2008. Vgl. hierzu noch H. Hamann, in diesem Heft, S. 102 f.

9 Dazu web.archive.org/20190304222500/iana.org/assignments/urn-namespaces/urn-namespaces.xh tml; das bereits erwähnte Auftakteditorial hatte bspw. die URN urn:nbn:de:0009-30-33650. 
Die ersten drei Jahre der Zeitschrift unter der Leitung der drei Gründungsherausgeber mündeten in drei veröffentlichte Jahrgänge: "Global Perspectives on the Language of Law" (2012), "Discourse in Law and its Language" (2013) und "Pragmatics and Discourse in Interpreting Law" (2014). Abgesehen vom bereits erwähnten Auftakteditorial, umfassten die Ausgaben 12 Beiträge mit insgesamt 180 Seiten, verfasst von Autoren aus Europa, Indien, Israel, Marokko, Mexiko, Südafrika und den Vereinigten Staaten. ${ }^{10}$

\section{Stillstand (2015): "Die Lücke, die Peter in der Wissenschaft hinterließ"}

Der 13. April 2014 wurde zum Schwarzen Sonntag für die ILLA und das JLL: Ihr Mitbegründer Peter Meijes Tiersma (geb. 23. Juni 1952) verstarb an Bauchspeicheldrüsenkrebs, im Alter von erst 61 Jahren. Geboren in den Niederlanden war Tiersma in den USA zum renommierten und angesehenen Rechtslinguisten avanciert, der zuletzt (seit 1990) eine Professur an der juristischen Fakultät der Marymount-Universität von Los Angeles (Loyola Law School) innehatte. Der Nachruf seiner Fakultät charakterisierte ihn als

„einen der führenden Rechtslinguisten der Nation. Nach einem Doktor in Sprachwissenschaften und einem juristischen Abschluss von der Universität Berkeley verfasste er zahlreiche herausragende Bücher und Artikel über die Beziehungen von Begriffen, linguistischen Medien, Bedeutung und Funktionsweise des Rechts. "11

Der vorzeitige Tod Tiersmas war verheerend für das noch junge JLL. Er hinterließ eine Lücke im Herausgeberkreis und unterbrach den Erscheinungsrhythmus der Zeitschrift, wie die späteren Herausgeber in einer Anmerkung zum Fehlen des vierten Jahrgangs erläuterten:

„Es gab keinen Jahrgang 4 des JLL. Die Zeitschrift blieb für ein Jahr unterbrochen, nachdem einer ihrer Gründer, Peter M. Tiersma (1952-2014), verstorben war. Das Ausbleiben des vierten Jahrgangs erinnert uns sehr sichtbar an die Lücke, die Peter in der Wissenschaft hinterließ, und an seine kritische Rolle beim JLL, der wir durch den offengelassenen vierten Jahrgang dankbar gedenken. "12

10 Autoren in alphabetischer Reihenfolge: E. Annamalai, Cornelis J. W. Baaij, Bill Bowring, Benjamin Brown, Bradly J. Condon, Lourens Marthinus Du Plessis, Elena Ioriatti, Fatima-Zahra Lamrani, Michael Moser, Anna Plisecka, Ronald Schmidt und Paulina Tallroth. [Ich bitte um Prüfung des Fußnotenankers: Im pdf-Korrekturabzug waren die beiden Ziffern übereinander abgedruckt.]

11 Siehe PeterTiersma.LLS.edu, und weiter: "He was a beloved teacher, winning the Best Professor of the Year Award several times. He was a member of many illustrious organizations, including the Oxford University Press editorial board for the law and language series, and advisory panels for both Black's Law Dictionary and the American Heritage Dictionary. He also served on the California Judicial Council Advisory Committee on Criminal Jury Instructions, where he helped rewrite California's jury instructions to make them more understandable and effective for jurors. He clerked for Justice Stanley Mosk of the California Supreme Court.”. [Ich bitte um Prüfung des Fußnotenankers: Im pdf-Korrekturabzug waren die beiden Ziffern übereinander abgedruckt.]

12 JLL Jg. 4: “Tribute to Peter M. Tiersma († 2014)”, unter web.archive.org/20190304225249/langua geandlaw.eu/jll/issue/view/4. 


\section{Die Wiederbelebung von ILLA und JLL (2016-2018): „Computergestützte Methoden, wo Text zu Daten wird"}

Das folgende Jahr schließlich, 2016, brachte einen Neuanfang für das JLL, und auchdessen Muttergesellschaft ILLA wurde ein Jahr später durch eine internationale Konferenz in Freiburg neubegründet. ${ }^{13}$ Beide Neuanfänge wurden von Prof. Dr. Friedemann Vogel orchestriert, damals in Freiburg, jetzt an der Universität Siegen. Er übernahm den Herausgeberposten des JLL (editor-in-chief), gründete ein Mitherausgebergremium renommierter Rechtslinguisten aus Wissenschaft und Praxis, ${ }^{14}$ und berief zum Schriftleiter (managing editor) den Autor des vorliegenden Textes als Jurist mit wissenschaftlichem Ausweis in der Rechtslinguistik und vormaliger Tätigkeit im Wissenschaftsverlag. Noch immer lebt die Zeitschrift vor allem vom ehrenamtlichen Engagement ihrer Herausgeber und Schriftleiter, wobei mittelfristig eine finanzielle und institutionelle Unterstützung durch die ILLA nicht ganz fernliegend erscheint.

Gemeinsam stellten der Herausgeber und der Schriftleiter die Zeitschrift vom DiPP-System auf das damals schon von knapp 10.000 Open-Access-Zeitschriften genutzte Open Journal System (OJS) des US-amerikanischen Bibliothekenverbunds Public Knowledge Project (PKP) um. ${ }^{15}$ Anstelle der bis dahin genutzten DPP-Lizenz kam fortan die international als Open-Access-Standard etablierte CreativeCommons-Lizenz CC BY 4.0 zum Einsatz. ${ }^{16}$ Als persistente Artikeladressen wurden die URNs durch die in der internationalen Wissenschaft verbreiteteren Digital Object Identifier (DOI) ersetzt. ${ }^{17}$ Diese bekommt das JLL dank Unterstützung des GESIS Leibniz-Instituts für Sozialwissenschaften von der deutschen Registrierungsagentur für Sozial- und Wirtschaftsdaten (dalra) kostenfrei zur Verfügung gestellt. ${ }^{18}$ Auch für die Langzeitarchivierung der JLL-Beiträge konnten laufende Kosten (wie sie insbesondere für CLOCKSS angefallen wären) vermieden werden, indem die Beiträge dem US-amerikanischen Datenbankanbieter HeinOnline für seine Law Journal Library (HOL) zur Verfügung gestellt wurden. ${ }^{19}$ Die anfangs noch angestrebte systematische Spiegelung von JLL-Beiträgen in einem nicht-kommerzi-

13 Siehe web.archive.org/20190304230255/illa.online/index.php/relaunch-conference-2017.

$14 \mathrm{Ihm}$ gehören neben den beiden überlebenden Gründern des JLL auch die Professoren R. Alexander Lorz, Łucja Biel, Dietrich Busse, Jan Engberg, Ekkehard Felder sowie Dr. Dr. Ralph Christensen an.

15 Vgl. web.archive.org/20190305221011/pkp.sfu.ca/ojs/ojs-usage/ojs-stats.

16 Dazu näher H. Hamann, in diesem Heft, S. 109.

17 Dazu web.archive.org/20190304231810/doi.org; die in Fn. 9 erwähnte URN wurde bspw. durch die DOI 10.14762/jll.2012.001 ersetzt.

18 Es bedarf hierzu eines einmaligen Vertragsschlusses mit GESIS, um sich als dalra-Nutzer zu registrieren (web.archive.org/20190305214639/da-ra.de/de/ueber-uns/unsere-nutzer). Die einzelnen DOIs können ohne weitere Rücksprache durch das DataCite-Plugin in OJS individuell registriert werden.

19 Unter web.archive.org/20190304230409/home.heinonline.org/titles/Law-Journal-Library/Internatio nal-Journal-of-Language--Law; auch hierzu bedurfte es eines einmaligen Vertragsschlusses, der aber angesichts der CC-BY-Lizensierung der Beiträge rein deklaratorisch sein dürfte. 
ellen Fachrepositorium wurde einstweilen zurückgestellt, weil alle einschlägigen Repositorien bislang entweder eine englischsprachige Benutzeroberfläche vermissen lassen (SSOAR, <intR $>$ 2Dok), oder keine belastbare Zukunftsprognose hinsichtlich der Nutzungsbereitschaft unter Rechtswissenschaftlern erlauben (SSRN, LawArXiv).

Abgesehen von solchen eher technischen Überlegungen „hinter den Kulissen“ gestaltete der Schriftleiter auch den öffentlichen Auftritt des JLL, dessen Emblem und Seitenlayout von Grund auf neu. Dabei wirkte die Open-Access-Verpflichtung bis in die Typographie hinein: Die Zeitschrift und ihre Website wurden in der als Open Font lizensierten und mehrfach ausgezeichneten Alegreya-Schriftfamilie des argentinischen Grafikdesigners Juan Pablo del Peral gesetzt. ${ }^{20}$

Abb. 2: Screenshot der JLL-Website auf der OJS-Plattform (seit 2016).

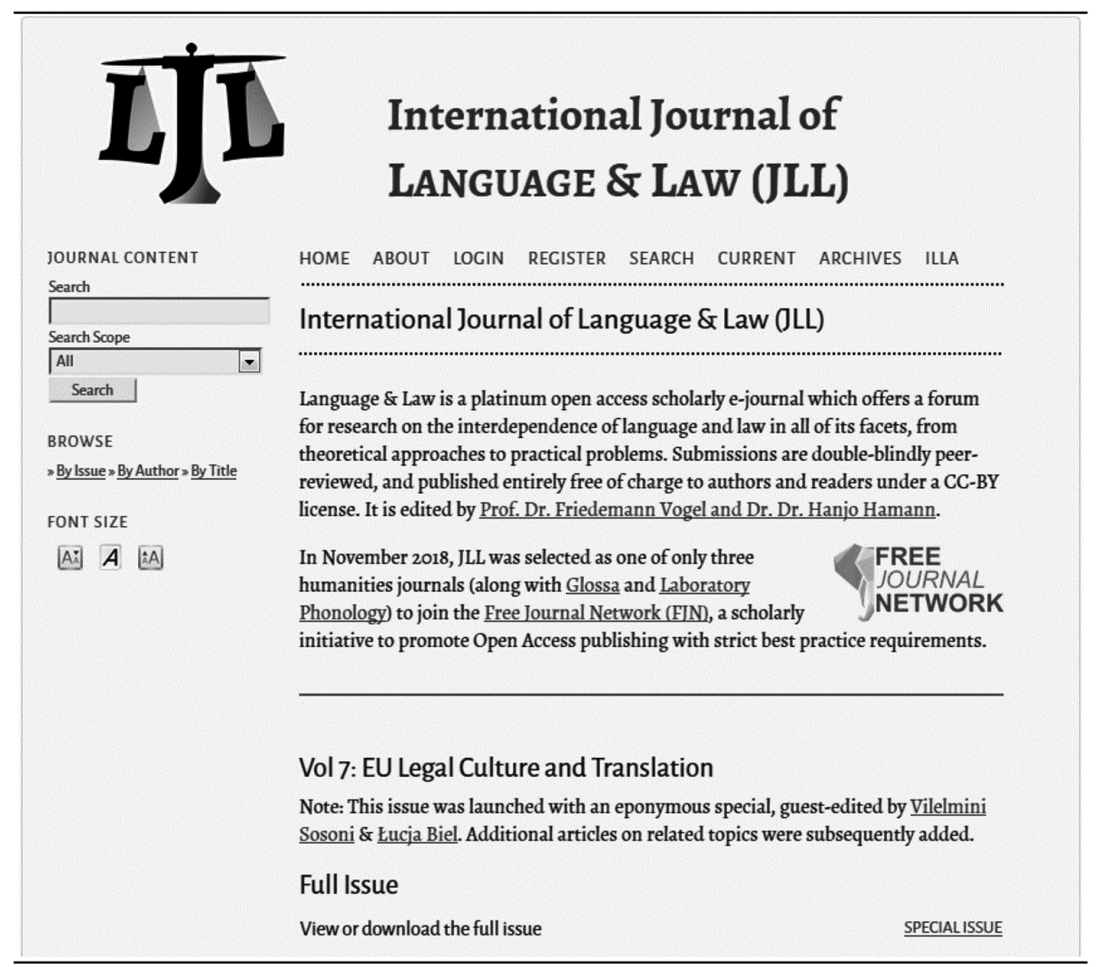

Screenshot vom 31. Dezember 2018, kurz vor dem zweiten Wechsel der Schriftleitung (dazu gleich Abschnitt E).

20 Zu Peral vgl. web.archive.org/20190305213227/huertatipografica.com/en/about, zur Open Font License (OFL 1.1) web.archive.org/20190305212951/scripts.sil.org/cms/scripts/page.php?site_id=nr si\&id=OFL\&_sc=1. 
Parallel zur Überarbeitung des Erscheinungsbildes wurden auch die Redaktionsrichtlinien der Zeitschrift überarbeitet oder erstmals ausformuliert. Diese betreffen insbesondere Fragen der Rechteeinräumung durch Autoren, Regeln der Kreuzbegutachtung (Peer Review) und Einhaltung guter wissenschaftlicher Praxis auf Basis der internationalen Empfehlungen des Committee on Publication Ethics (COPE). ${ }^{21}$

Inhaltlich resultierte das zweite Triennium der Zeitschrift erneut in drei veröffentlichten Jahrgängen: "International Law and Pragmatics" (2016), "The Fabric of Language and Law" (2017) sowie "EU Legal Culture and Translation" (2018). Die zweite dieser Ausgaben beruhte auf einer internationalen Tagung in Heidelberg, ${ }^{22}$ die angeregt worden war durch den

"fundamentalen Wandel des Kontextes von Sprache und Recht während der letzten zwanzig Jahre. Die Digitalisierung aller Bereiche des Lebens verändert die Texturen des Rechts und die Muster unserer Sprache. Ihre tieferliegenden Fasern lassen sich leichter erkennen und erkunden: Intertextualität, Verweise, usw. sind nun , anklickbar im Hypertext und seinen Medien. [...] Dieser digitale Trend eröffnet neues Potential für die Rechtslinguistik: Sie kann computergestützte Methoden entwickeln, wo Text zu Daten wird. "23

$\mathrm{Zu}$ einem Aspekt dieses Themas durfte das JLL auch erstmals einen seiner Gründungsherausgeber als Autor begrüßen. ${ }^{24} \mathrm{Im}$ Jahr darauf lud das JLL erneut Gastherausgeber ein, wie schon fünf Jahre zuvor (siehe oben, Abschnitt B): Der Hauptteil des Jahrgangs wurde konzipiert und die Peer Review organisiert von Vilelmini Sosoni (Ionische Universität Korfu) und Lucja Biel (Universität Warschau). Ihr Sonderheft erschien im Nachgang zur Konferenz, mit der die ILLA neugegründet worden war, ${ }^{25}$ die Herausgeberinnen eröffneten es mit einer Zusammenfassung der

„fünf Beiträge von Juristinnen und Sprachwissenschaftlerinnen, die die Komplexität und Hybridität der Rechts- und Übersetzungskultur der Europäischen Union aus einer Reibe von theoretischen und methodischen Perspektiven beleuchten. Das Thema wird aus verschiedenen Blickrichtungen betrachtet, um quantitative und qualitative Methoden (Rechts-

21 Alle Richtlinien unter web.archive.org/20190304231601/languageandlaw.eu/jll/about/editorialPolic ies.

22 Dazu Ch. Lukas, Korpuslinguistik und Recht. Bericht über die Konferenz „The Fabric of Law and Language“ der Heidelberger Akademie der Wissenschaften vom 18. und 19. März 2016, ARSP 2017, S. 136 (138), sowie Vogel/Hamann u.a., Lawyers and Linguists (Fn. 2).

23 H. Hamann/F. Vogel, The Fabric of Language and Law. Towards an International Research Network for Computer Assisted Legal Linguistics (CAL 2 ), JLL 2017, S. 101 (103f.), abrufbar unter doi.org/10.14762/jll.2017.101.

24 L. M. Solan, Patterns in Language and Law, JLL 2017, S. 46, abrufbar unter doi.org/10.14762/ jll.2017.046.

25 Siehe Fußnote 13 oben, sowie V. Sosoni/L. Biel, EU Legal Culture and Translation, JLL 2018, S. 1 (2): "This special issue [...] is based on the panel organised by the authors at the Language and Law in a World of Media, Globalisation and Social Conflicts conference (7-9 September 2017, University of Freiburg, Germany), which relaunched the International Law and Language Association (ILLA).”. 
vergleichung, Rechtstheorie und Logik, Korpuslinguistik und kritische Diskursanalyse) miteinander $z$ u triangulieren. " 26

Zusammengenommen enthalten die drei Jahrgänge 2016-2018 des JLL 15 Beiträge mit 315 Seiten, verfasst von 23 verschiedenen Autoren ${ }^{27}$ aus sechs Kontinenten. ${ }^{28}$ Als weitere Kennzahlen für diesen Zeitraum ergaben sich eine durchschnittliche Wartezeit von der Beitragseinreichung bis zur redaktionellen Entscheidung (turn around) von 52,3 Tagen, bis zu 94 Tage im längsten Begutachtungsverfahren. Gut die Hälfte $(53 \%)$ der eingereichten Beiträge wurden abgelehnt, von diesen wiederum zwei Fünftel (20\% von allen) durch die Schriftleitung ohne externe Begutachtung (desk rejection). Von den zur Begutachtung ausgesandten Beiträgen wurden $75 \%$ von je einem Juristen und einem Sprachwissenschaftler begutachtet.

Nicht zuletzt aufgrund der hohen Qualitätsansprüche, die ihre Herausgeber an die Zeitschrift stellten, wurde das JLL im November 2018 als eine von nur drei geisteswissenschaftlichen Zeitschriften (neben Glossa und Laboratory Phonology) ${ }^{29}$ zum Mitglied des Free Journal Network (FJN) gewählt, ${ }^{30}$ einer Initiative von Wissenschaftlern zur Etablierung und Förderung höchster Praxisstandards (best practices) im Open-Access-Publikationswesen.

\section{E. Zukunftsperspektiven der Zeitschrift (2019-2021)}

$\mathrm{Zu}$ Beginn ihres dritten Trienniums erfreut sich die Zeitschrift einer beachtlichen Bekanntheit über Fachgebietsgrenzen hinweg. Sie findet sich in so unterschiedlichen Disziplinen zitiert wie Literatur- und Kulturwissenschaften, ${ }^{31}$ Rhetorikstudi-

26 V. Sosoni/L. Biel, EU Legal Culture and Translation, JLL 2018, S. 1 (4), abrufbar unter doi.org/ 10.14762/j1l.2018.001.

27 Autoren in alpgabetischer Reihenfolge: Andreas Abegg, Martina Bajčić, Łucja Biel (2×), Ruth Breeze, John Burke, Aleksandra Čavoški, Christoph A. Hafner, Hanjo Hamann (2×), Anna Jopek-Bosiacka, Sofiya Kartalova, Elpida Loupaki, Katherine Lynch, María José Marín, Stephen Mouritsen, Benedikt Pirker (2x), Anne Scully-Hill, Rajesh Sharma, Jennifer Smolka (2x), Lawrence M. Solan (2x), Vilelmini Sosoni, Dieter Stein und Friedemann Vogel $(2 \times)$.

28 Einreichungen stammten aus Albanien, Australien, Österreich, China/Hongkong, Kolumbien, Koratien, Deutschland, Griechenland, Italien, Kenia, Nigeria, Polen, Spanien, der Schweiz, dem Vereinigten Königreich, und den USA.

29 Erreichbar unter web.archive.org/20190304234042/glossa-journal.org bzw. web.archive.org/20190 304233937/journal-labphon.org.

30 web.archive.org/20190304234112/freejournals.org.

31 T. Jati Murtaya/S. Triyono, Omission in Possible: The Forensic Linguistics Autopsy of the Court Interpreting Praxis, International Journal of Comparative Literature \& Translation Studies 6 (2018), S. 50 (52); V. M. Chanda, A Bird's Eye View of 'Language and Law', International Journal of Humanities and Cultural Studies 4 (2018), S. 32 (33). 
en, ${ }^{32}$ Sprachpolitik, ${ }^{33}$ Rechtsphilosophie ${ }^{34}$ und Rechtswissenschaften ${ }^{35}$ - bis in so exotische Gebiete wie das tschechische Waffenrecht. ${ }^{36}$

Um dieses Ansehen weiter auszubauen, wurde zum Jahresbeginn 2019 der Staffelstab erneut weitergegeben: Neuer Schriftleiter ist Dr. Aleksandar Trklja, ein in vergleichender Korpuslinguistik (Deutsch/Englisch) promovierter Sprachwissenschaftler mit Forschungserfahrung im Bereich der EuGH-Rechtsprechung, ${ }^{37}$ der aktuell an der Universität Wien lehrt. Parallel zur Ernennung dieses ersten nicht aus Deutschland stammenden Schriftleiters ist die Zeitschrift von ihrer bisherigen Domain LanguageAndLaw.de zum europäischen Pendant LanguageAndLaw.eu umgezogen, um die weitere Internationalisierung und breitere regionale Perspektive zu dokumentieren, die von Trkljas Schriftleiterschaft ausgehen wird. Unter seiner Leitung wird das JLL in der näheren Zukunft auch die Aufnahme in das internationale Directory of Open Access Journals (DOAJ, doaj.org) anstreben, die bisher durch das DOAJ-Reglement wegen des im Jahr 2015 unterbrochenen Erscheinungsrhythmus' verhindert wurde.

Auch inhaltlich lag wieder ein spannendes Jahr vor der Zeitschrift, die im Februar 2019 ihren diesjährigen Beitragsaufruf zum Jahresthema "The Impact of Language on Law-Making and Norm-Making" veröffentlicht hat. ${ }^{38}$ Mit der Rolle von Sprache in Normgenese und Gesetzgebung widmet sich das JLL einem aktuell noch immer unterschätzten und zu selten wissenschaftlich bearbeiteten Forschungsdesiderat. Der Autor, der auch nach Ende seiner Schriftleitertätigkeit dem JLL als Herausgeber verbunden bleibt, sieht der weiteren Entwicklung der Rechtslinguistik ebenso gespannt entgegen wie der weiteren Aufnahme, die dem JLL als Open-Access-Zeitschrift in der Fachgemeinschaft widerfährt.

32 O. Łachacz/R. Mańko, Multilingualism at the Court of Justice of the European Union, Studies in Logic, Grammar and Rhetoric 34 (2013), S. 75 (80); R. Mańko, Multilingualism, Divergent Authentic Versions of a Legal Rule and Legitimate Expectations of Individuals, Studies in Logic, Grammar and Rhetoric 45 (2016), S. 141 (142).

33 M. De Meulder, Promotion in times of endangerment: The Sign Language Act in Finland, Language Policy, 16 (2017), S. 189 (193, 195, 203).

34 F. Macagno/D. Walton/G. Sartor, Pragmatic Maxims and Presumptions in Legal Interpretation, Law and Philosophy 37 (2018), S. 69 (69, 72); D. Walton/G. Sartor/F. Macagno, Statutory Interpretation as Argumentation. In Bongiovanni, Postema u.a. (Hrsg.), Handbook of Legal Reasoning and Argumentation 2018, S. 519 (ebd.).

35 D. Tankersley, Beyond the Dictionary: Why SUA Sponte Judicial Use of Corpus Linguistics Is Not Appropriate for Statutory Interpretation, Mississippi Law Journal 87 (2018), S. 641 (671 Fn. 164).

36 K. Williams, Keeping and Bearing Arms in Czech, In Fidler \& Cvrček (Hrsg.), Taming the Corpus: From Inflection and Lexis to Interpretation 2018, S. 148 (ebd.).

37 A. Trklia, A Corpus linguistics study of translation correspondences in English and German, University of Birmingham School of English doctoral thesis 2013, abrufbar unter web.archive.org/201903 04233543/etheses.bham.ac.uk/id/eprint/4785/1/Trklja14PhD.pdf. Trklja war zuvor im ERCForschungsprojekt "The Law and Language at the European Court of Justice” beschäftigt: web.arc hive.org/20190305211330/llecj.karenmcauliffe.com/the-team.

38 A. Trklja, The Impact of Language on Law-Making and Norm-Making: JLL Call for Papers (Deadline 31 July 2019), JLL 2019, S. 9, abrufbar unter doi.org/10.14762/j1l.2019.009. 